\title{
The Panpositionable Pancyclicity of Locally Twisted Cubes
}

\author{
Hon-Chan CHEN ${ }^{\dagger a)}$, Member
}

SUMMARY In a multiprocessor system, processors are connected based on various types of network topologies. A network topology is usually represented by a graph. Let $G$ be a graph and $u, v$ be any two distinct vertices of $G$. We say that $G$ is pancyclic if $G$ has a cycle $C$ of every length $l(C)$ satisfying $3 \leq l(C) \leq|V(G)|$, where $|V(G)|$ denotes the total number of vertices in $G$. Moreover, $G$ is panpositionably pancyclic from $r$ if for any integer $m$ satisfying $r \leq m \leq \frac{|V(G)|}{2}, G$ has a cycle $C$ containing $u$ and $v$ such that $d_{C}(u, v)=m$ and $2 m \leq l(C) \leq|V(G)|$, where $d_{C}(u, v)$ denotes the distance of $u$ and $v$ in $C$. In this paper, we investigate the panpositionable pancyclicity problem with respect to the $n$-dimensional locally twisted cube $L T Q_{n}$, which is a popular topology derived from the hypercube. Let $D\left(L T Q_{n}\right)$ denote the diameter of $L T Q_{n}$. We show that for $n \geq 4$ and for any integer $m$ satisfying $D\left(L T Q_{n}\right)+2 \leq m \leq \frac{\left|V\left(L T Q_{n}\right)\right|}{2}$, there exists a cycle $C$ of $L T Q_{n}$ such that $d_{C}(u, v)=m$, where (i) $2 m+1 \leq l(C) \leq\left|V\left(L T Q_{n}\right)\right|$ if $m=D\left(L T Q_{n}\right)+2$ and $n$ is odd, and (ii) $2 m \leq l(C) \leq\left|V\left(L T Q_{n}\right)\right|$ otherwise. This improves on the recent result that $u$ and $v$ can be positioned with a given distance on $C$ only under the condition that $l(C)=\left|V\left(L T Q_{n}\right)\right|$. In parallel and distributed computing, if cycles of different lengths can be embedded, we can adjust the number of simulated processors and increase the flexibility of demand. This paper demonstrates that in $L T Q_{n}$, the cycle embedding containing any two distinct vertices with a feasible distance is extremely flexible.

key words: cycle embedding, pancyclicity, locally twisted cube, interconnection network

\section{Introduction}

In a multiprocessor system, processors are connected based on various types of network topologies. A network topology is usually represented by a graph, in which the vertices and edges of the graph represent the processors and communication links of the multiprocessor system. In this paper, we use an undirected graph to represent a network topology, and we follow the graph-theory definitions and notations given by Golumbic [6].

Let $G=(V, E)$ be an undirected graph with vertex set $V(G)$ and edge set $E(G)$. We use $|V(G)|$ to denote the number of vertices in $G$, and we use $u v$ to denote the edge incident with vertices $u$ and $v$. Two vertices $u$ and $v$ of $G$ are adjacent if $u v \in E(G)$. A path $P$ of $G$ is a sequence of distinct vertices such that any two consecutive vertices of $P$ are adjacent in $G$. The length of $P$, denoted by $l(P)$, is the number of edges in $P$. For a path $P$ of length $k, k \geq 1$, we write

\section{Manuscript received December 29, 2017.}

Manuscript revised April 27, 2018.

Manuscript publicized September 18, 2018.

${ }^{\dagger}$ The author is with the Department of Information Management, National Chin-Yi University of Technology, Taiping, Taichung City 411, Taiwan.

a)E-mail: chenhc@ncut.edu.tw

DOI: 10.1587/transinf.2018PAP0006
$P=\left[v_{1}, v_{2}, \ldots, v_{k}, v_{k+1}\right]$. The $i$ th vertex of $P$ is denoted by $P(i)$. If $P^{*}=\left[v_{i}, \ldots, v_{j}\right], i \leq j$, is a segment of $P$, we can also write $P$ as $\left[v_{1}, v_{2}, \ldots, v_{i}, P^{*}, v_{j}, \ldots, v_{k+1}\right]$. A cycle is a close path with at least three vertices, where the last vertex is adjacent to the first one.

The distance between vertices $u$ and $v$ in $G$, denoted by $d_{G}(u, v)$, is the length of the shortest path between $u$ and $v$. The diameter of $G$, denoted by $D(G)$, is the maximum of all distances for all pairs of vertices in $G$. A graph $H$ is a subgraph of $G$ if $V(H) \subseteq V(G)$ and $E(H) \subseteq E(G)$. If $V(H)=V(G), H$ is a spanning subgraph of $G$, or we can say that $H$ spans $G$. A Hamiltonian path (respectively, Hamiltonian cycle) of $G$ is a path (respectively, cycle) which spans $G$. A graph $G$ is Hamiltonian if it has a Hamiltonian cycle, and $G$ is Hamiltonian connected if it has a Hamiltonian path joining any pair of vertices of $G$.

Let $u$ and $v$ be any two distinct vertices of $G$. We say that $G$ is pancyclic if it has a cycle of every length $m$ satisfying $3 \leq m \leq|V(G)|[2]$. More specifically, $G$ is vertex-pancyclic (respectively, edge-pancyclic) if its any vertex (respectively, edge) lies on a cycle of every length $m, 3 \leq m \leq|V(G)|$. On the other hand, $G$ is panconnected if it has a path of every length $m$ joining $u$ and $v$ with $d_{G}(u, v) \leq m \leq|V(G)|-1$ for any two vertices $u$ and $v$ of $G$ [1]. If there exists a Hamiltonian cycle $C$ of $G$ such that $d_{C}(u, v)=m$ for any integer $m$ with $d_{G}(u, v) \leq m \leq$ $\frac{|V(G)|}{2}$, then $G$ is panpositionably Hamiltonian [14]. From the above definitions, we can further define that a graph $G$ is panpositionably pancyclic if it has a cycle $C$ containing $u$ and $v$ such that (i) $d_{C}(u, v)=m$ for any integer $m$ with $d_{G}(u, v) \leq m \leq \frac{|V(G)|}{2}$, and (ii) $2 m \leq l(C) \leq|V(G)|$ for every $l(C)$; i.e., vertices $u$ and $v$ can be positioned on various cycles $C$ of feasible lengths in $G$ such that $u$ and $v$ keep any given distance from each other on $C$. In this paper, we will investigate the panpositionable pancyclicity problem with respect to locally twisted cubes.

The locally twisted cube was introduced by Yang et al. [16]. It can be derived from the hypercube by changing half part of links. An $n$-dimensional locally twisted cube, denoted by $L T Q_{n}$, has $2^{n}$ vertices, and each vertex corresponds to an $n$-bit binary string. The locally twisted cube is recursively constructed as follows:

(i) $L T Q_{2}$ is a graph with four vertices $w=00, x=01, y=$ $10, z=11$, and the edge set of $L T Q_{2}$ is $\{w x, w y, x z, y z\}$.

(ii) For $n \geq 3$, let $L T Q_{n-1}^{0}$ and $L T Q_{n-1}^{1}$ be two copies of $L T Q_{n-1}$ with $V\left(L T Q_{n-1}^{0}\right)=\left\{0 u_{n-1} u_{n-2} \ldots u_{1} \mid u_{i}=\right.$ 


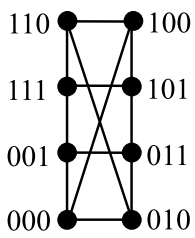

$\mathrm{LTQ}_{3}$

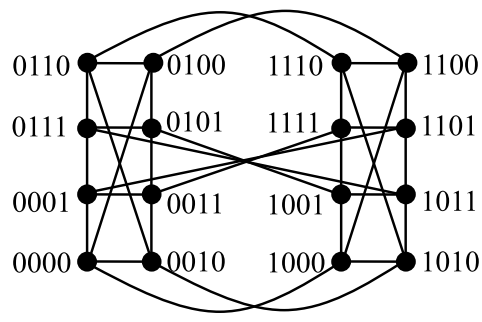

$\mathrm{LTQ}_{4}$
Fig. $1 \quad L T Q_{3}$ and $L T Q_{4}$.

0 or 1 for $1 \leq i \leq n-1\}$ and $V\left(L T Q_{n-1}^{1}\right)=$ $\left\{1 u_{n-1} u_{n-2} \ldots u_{1} \mid u_{i}=0\right.$ or 1 for $\left.1 \leq i \leq n-1\right\}$. Then, $L T Q_{n}$ is formed by connecting $L T Q_{n-1}^{0}$ and $L T Q_{n-1}^{1}$ with $2^{n-1}$ edges such that a vertex $u=0 u_{n-1} u_{n-2} \ldots u_{1}$ in $L T Q_{n-1}^{0}$ is adjacent to a vertex $v=1 v_{n-1} v_{n-2} \ldots v_{1}$ in $L T Q_{n-1}^{1}$ if and only if $v_{n-1}=\left(u_{n-1}+u_{1}\right) \bmod 2$ and $v_{i}=u_{i}$ for every $i, 1 \leq i \leq n-2$.

Figure 1 shows the $L T Q_{3}$ and $L T Q_{4}$. It is proven that the diameter $D\left(L T Q_{n}\right)$ is $n-1$ if $n \in\{3,4\}$ and $D\left(L T Q_{n}\right)$ is $\left\lceil\frac{n+3}{2}\right\rceil$ if $n \geq 5$, which is about half that of the hypercube [16].

According to its definition and structure, a locally twisted cube has no cycle of length 3 . That is, for any edge $u v$ of $L T Q_{n}$, there exists no path of length 2 joining $u$ and $v$ in $L T Q_{n}$. Therefore, locally twisted cubes are not fully pancyclic or panconnected. This property also can be found in several hypercube variants. Many researchers then proposed loosed versions of pancyclicity and panconnectedness for various cubes [3], [5], [10], [12]. Generally, a graph $G$ is defined to be r-pancyclic if it has a cycle of every length $m$ satisfying $r \leq m \leq|V(G)|$, and $G$ is defined to be $r$-panconnected if it has a path of every length $m$, $r \leq m \leq|V(G)|-1$, joining $u$ and $v$ for any two distinct vertices $u$ and $v$ of $G$.

In [17], Yang et al. showed that locally twisted cubes are 4-pancyclic and Hamiltonian connected. Hu et al. further showed that locally twisted cubes are 4-node-pancyclic and 4-edge-pancyclic [7]. In [12], Ma and Xu proposed the panconnectedness of locally twisted cubes, where for any two distinct vertices $u$ and $v$ of $L T Q_{n}, n \geq 3$, there exists a path of every length $m$ joining $u$ and $v$ with $d_{L T Q_{n}}(u, v) \leq$ $m \leq 2^{n}-1$ and $m \neq d_{L T Q_{n}}(u, v)+1$. Kung and Chen in [9] combined panconnectedness with Hamiltonian connectedness to show that the $n$-dimensional locally twisted cube is Hamiltonian $n$-panconnected for $n \geq 5$. More precisely, for any three distinct vertices $x, y$, and $z$ of $L T Q_{n}$, there exists a Hamiltonian path $P$ of $L T Q_{n}$ such that $P(1)=x$, $P(m+1)=y$, and $P\left(2^{n}\right)=z$ for any integer $m$ satisfying $n \leq m \leq 2^{n}-n-1$. In [8], Kung discussed the panpositionable Hamiltonicity of locally twisted cubes. He showed that for any two distinct vertices $u$ and $v$ of $L T Q_{n}, n \geq 4$, and for any integer $m$ with $d_{L T Q_{n}}(u, v) \leq m \leq 2^{n-1}$ and $m \neq d_{L T Q_{n}}(u, v)+1$, there exists a Hamiltonian cycle $C$ of $L T Q_{n}$ such that $d_{C}(u, v)=m$. In this paper, we will improve the above result of panpositionable Hamiltonicity so that the cycle $C$ of $L T Q_{n}$ can be of various feasible lengths instead of a Hamiltonian cycle.

The rest of this paper is organized as follows. In Sect. 2, we introduce some properties of locally twisted cubes. In Sect. 3, we propose the main result of this paper and prove its correctness. Finally, some concluding remarks are given in Sect. 4.

\section{Preliminaries}

Let $u=u_{n} u_{n-1} \ldots u_{1}$ and $v=v_{n} v_{n-1} \ldots v_{1}$ be any two distinct vertices of $L T Q_{n}$. Then, $u$ and $v$ are adjacent along the $i$ th dimension, $1 \leq i \leq n$, if and only if one of the following conditions is satisfied [17]:

(i) For $i \in\{1,2\}$, vertices $u$ and $v$ differ only with $u_{i} \neq v_{i}$.

(ii) For $3 \leq i \leq n$, vertices $u$ and $v$ are with (a) $u_{i} \neq v_{i}$, (b) $u_{i-1}=\left(v_{i-1}+u_{1}\right) \bmod 2$, and (c) $u_{j}=v_{j}$ for all $j \in\{1,2, \ldots, n\}-\{i-1, i\}$.

For convenience, we say that $v$ is the $i$-neighbor of $u$, and it is denoted by $v=(u)^{i}$. We also call that $u v$ is an $i$-dimensional edge. The following lemmas describe some properties of cycle embedding and path embedding of locally twisted cubes.

Lemma 1: [9] Let $u v$ be any $n$-dimensional edge of $L T Q_{n}$, $n \geq 3$. Then, vertices $u, v,(u)^{i}$, and $(v)^{i}$ form a cycle of length 4 if $2 \leq i \leq n-1$.

Lemma 2: [8] Let $u$ and $v$ be any two distinct vertices of $L T Q_{n}, n \geq 2$, where $u$ is in $L T Q_{n-1}^{0}$ and $v$ is in $L T Q_{n-1}^{1}$. Then, $d_{L T Q_{n}}\left(u,(v)^{n}\right)=d_{L T Q_{n}}(u, v)-1$ or $d_{L T Q_{n}}\left((u)^{n}, v\right)=$ $d_{L T Q_{n}}(u, v)-1$.

Lemma 3: [12] Let $u$ and $v$ be any two distinct vertices of $L T Q_{n}, n \geq 3$. For any integer $m$ satisfying $d_{L T Q_{n}}(u, v) \leq$ $m \leq 2^{n}-1$ and $m \neq d_{L T Q_{n}}(u, v)+1$, there exists a path $P$ of $L T Q_{n}$ joining $u$ and $v$ such that $l(P)=m$.

Lemma 4: [8] Let $u$ and $v$ be any two distinct vertices of $L T Q_{n}, n \geq 4$. For any integer $m$ satisfying $d_{L T Q_{n}}(u, v) \leq m \leq$ $2^{n}-1$ and $m \neq d_{L T Q_{n}}(u, v)+1$, there exists a Hamiltonian path $P$ of $L T Q_{n}$ such that $P(1)=u$ and $P(m+1)=v$; i.e., $d_{P}(u, v)=m$.

Let $G$ be a graph and $F$ be a set of vertices and/or edges of $G$. We use $G-F$ to denote the resulting graph after removing the elements of $F$ from $G$. Then, a Hamiltonian graph $G$ is $f$-fault-tolerant Hamiltonian (or $f$-Hamiltonian) if $G-F$ remains Hamiltonian for every $F$ with $|F| \leq f$. Similarly, a Hamiltonian connected graph $G$ is $f$-fault-tolerant Hamiltonian connected (or $f$-Hamiltonian connected) if $G-F$ remains Hamiltonian connected for every $F$ with $|F| \leq f$. The following lemmas give some properties of fault-tolerance for locally twisted cubes.

Lemma 5: [13] Locally twisted cubes $L T Q_{n}, n \geq 3$, are $(n-2)$-Hamiltonian and $(n-3)$-Hamiltonian connected.

Lemma 6: [15] Let $F$ be a set of faulty vertices and/or edges in $L T Q_{n}$, where $n \geq 3$ and $|F| \leq n-3$. Moreover, let $u v$ be any edge of $L T Q_{n}-F$. Then, there exists a path 


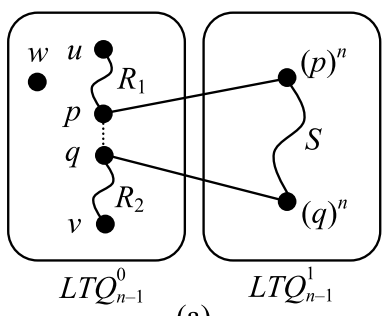

(a)

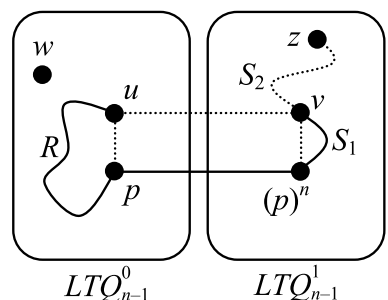

(b)

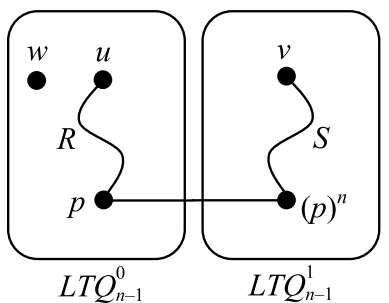

(c)

Fig. 2 Illustration of the proof of Lemma 7. (A straight line represents an edge, and a curve represents a path.)

$P$ of $L T Q_{n}-F$ joining $u$ and $v$ such that $l(P)=m$ for every integer $m$ satisfying $5 \leq m \leq 2^{n}-|F|-1$.

Lemma 7: Let $w$ be any vertex of $L T Q_{n}, n \geq 4$. Moreover, let $u$ and $v$ be any two distinct vertices of $L T Q_{n}-\{w\}$. Then, there exists a path $P$ of $L T Q_{n}-\{w\}$ joining $u$ and $v$ such that $l(P)=m$ for every integer $m$ satisfying $\left\lceil\frac{n+3}{2}\right\rceil+3 \leq m \leq$ $2^{n}-2$

Proof. We prove this lemma by induction. The correctness of the induction base $L T Q_{4}$ can be verified by our computer program [4]. Assume, as the inductive hypothesis, that this lemma holds for any $L T Q_{k}, 4 \leq k \leq n-1$. We will show that this lemma also holds for $L T Q_{n}$. Without loss of generality, assume $w$ is in $L T Q_{n-1}^{0}$. Moreover, assume $u$ is in $L T Q_{n-1}^{0}$ if $u$ and $v$ are respectively in different subcubes $L T Q_{n-1}^{i}$ and $L T Q_{n-1}^{1-i}$ of $L T Q_{n}, i \in\{0,1\}$. According to the possibilities of $u$ and $v$, we distinguish the following cases.

Case 1. $u$ and $v$ are in $L T Q_{n-1}^{0}$. In this case, we also need to consider the following subcases.

Subcase 1.1. $\left\lceil\frac{n+3}{2}\right\rceil+3 \leq m \leq 2^{n-1}-2$. By the inductive hypothesis, there exists a path $R$ of $L T Q_{n-1}^{0}-\{w\}$ joining $u$ and $v$ with $\left\lceil\frac{(n-1)+3}{2}\right\rceil+3 \leq l(R) \leq 2^{n-1}-2$. Certainly, this path is a path of $L T Q_{n}-\{w\}$ and it also satisfies $\left\lceil\frac{n+3}{2}\right\rceil+3 \leq$ $l(R) \leq 2^{n-1}-2$. We let $P=R$, and $P$ is our required path.

Subcase 1.2. $2^{n-1}-1 \leq m \leq 2^{n}-2$. By the inductive hypothesis, $L T Q_{n-1}^{0}-\{w\}$ has a path $R$ joining $u$ and $v$ with $2^{n-2}-1 \leq l(R) \leq 2^{n-1}-2$. We write $R$ as $\left[u, R_{1}, p, q, R_{2}, v\right]$ for some adjacent vertices $p$ and $q$. By Lemma $3, L T Q_{n-1}^{1}$ has a path $S$ joining $(p)^{n}$ and $(q)^{n}$ with $2^{n-2}-1 \leq l(S) \leq 2^{n-1}-1$. We set $P=\left[u, R_{1}, p,(p)^{n}, S,(q)^{n}, q, R_{2}, v\right]$, then $P$ is a path of $L T Q_{n}-\{w\}$ joining $u$ and $v$ with $l(P)=l(R)+l(S)+1$. It is easy to obtain that $2^{n-1}-1 \leq l(P) \leq 2^{n}-2$, and $l(P)=m$. See Fig. 2 (a) for illustration.

Case 2. $u$ and $v$ are in $L T Q_{n-1}^{1}$. In this case, two subcases are distinguished.

Subcase 2.1. $\left\lceil\frac{n+3}{2}\right\rceil+3 \leq m \leq 2^{n-1}-1$. By Lemma 3, we have a path $S$ of $L T Q_{n-1}^{1}$ joining $u$ and $v$ with $\left\lceil\frac{(n-1)+3}{2}\right\rceil+2 \leq$ $l(S) \leq 2^{n-1}-1$, which also satisfies $\left\lceil\frac{n+3}{2}\right\rceil+3 \leq l(S) \leq$ $2^{n-1}-1$. We can let $P=S$.

Subcase 2.2. $2^{n-1} \leq m \leq 2^{n}-2$. In $L T Q_{n-1}^{1}$, by Lemma 3, there exists a path $S$ joining $u$ and $v$ with $2^{n-2}-1 \leq$ $l(S) \leq 2^{n-1}-1$. We can write $S$ as $\left[u, S_{1}, p, q, S_{2}, v\right]$ for some adjacent vertices $p$ and $q$ with $(w)^{n} \notin\{p, q\}$. By the inductive hypothesis, $L T Q_{n-1}^{0}-\{w\}$ has a path $R$ join- ing $(p)^{n}$ and $(q)^{n}$ with $2^{n-2} \leq l(R) \leq 2^{n-1}-2$. We set $P=\left[u, S_{1}, p,(p)^{n}, R,(q)^{n}, q, S_{2}, v\right]$, and $l(P)=l(R)+l(S)+1$. Then, we have that $2^{n-1} \leq l(P) \leq 2^{n}-2$, and $l(P)=m$.

Case 3. $u$ is in $L T Q_{n-1}^{0}$ and $v$ is in $L T Q_{n-1}^{1}$ and $u v$ is an edge of $L T Q_{n}$. By Lemma 1, we can find a cycle of length 4 that contains $u$ and $v$ and does not contain $w$. We write this cycle as $\left[u, p,(p)^{n}, v, u\right]$. By the inductive hypothesis, there exists a path $R$ in $L T Q_{n-1}^{0}-\{w\}$ joining $u$ and $p$ with $l(R) \in$ $\left\{1,\left\lceil\frac{n+2}{2}\right\rceil+3,\left\lceil\frac{n+2}{2}\right\rceil+4, \ldots, 2^{n-1}-2\right\}$. In $L T Q_{n-1}^{1}$, Lemma 4 ensures that there exists a Hamiltonian path $S$ such that $S(1)=(p)^{n}$ and $d_{S}\left((p)^{n}, v\right) \in\left\{1,3,4, \ldots, 2^{n-1}-1\right\}$. We can write $S=\left[(p)^{n}, S_{1}, v, S_{2}, z\right]$ for some vertex $z$ of $L T Q_{n-1}^{1}$. Note that $v=z$ if $l\left(S_{2}\right)=0$. We set $P=\left[u, R, p,(p)^{n}, S_{1}, v\right]$, and $l(P)=l(R)+l\left(S_{1}\right)+1$. It is obvious that $l(P)=3$ or $5 \leq l(P) \leq 2^{n}-2$. Since $\left\lceil\frac{n+3}{2}\right\rceil+3>5$ for $n \geq 5, l(P)$ satisfies $\left\lceil\frac{n+3}{2}\right\rceil+3 \leq l(P) \leq 2^{n}-2$, and $P$ can be our required path. See Fig. 2 (b) for illustration.

Case 4. $u$ is in $L T Q_{n-1}^{0}$ and $v$ is in $L T Q_{n-1}^{1}$ and $u$ is not adjacent to $v$. In this case, we need to consider the following two subcases.

Subcase 4.1. $\left\lceil\frac{n+3}{2}\right\rceil+3 \leq m \leq 2^{n-1}$. By Lemma 3, $L T Q_{n-1}^{1}$ has a path $S$ joining $(u)^{n}$ and $v$ with $\left\lceil\frac{n+2}{2}\right\rceil+2 \leq l(S) \leq$ $2^{n-1}-1$. We set $P=\left[u,(u)^{n}, S, v\right]$, and $l(P)=l(S)+1$, which satisfies $\left\lceil\frac{n+3}{2}\right\rceil+3 \leq l(P) \leq 2^{n-1}$. Thus, $P$ can be our required path.

Subcase 4.2. $2^{n-1}+1 \leq m \leq 2^{n}-2$. Let $p$ be a vertex of $L T Q_{n-1}^{0}-\{w\}$ with $(p)^{n} \neq v$. By the inductive hypothesis, $L T Q_{n-1}^{0}-\{w\}$ has a path $R$ joining $u$ and $p$ with $2^{n-2} \leq l(R) \leq$ $2^{n-1}-2$. By Lemma 3, LT $Q_{n-1}^{1}$ has a path $S$ joining $(p)^{n}$ and $v$ with $2^{n-2} \leq l(S) \leq 2^{n-1}-1$. We set $P=\left[u, R, p,(p)^{n}, S, v\right]$, and $l(P)=l(R)+l(S)+1$. Then, we have that $2^{n-1}+1 \leq$ $l(P) \leq 2^{n}-2$, and $l(P)=m$. See Fig. 2 (c) for illustration.

The above argument completes the proof.

\section{The Panpositionable Pancyclicity Problem}

In this section, we propose the panpositionable pancyclicity problem of locally twisted cubes $L T Q_{n}$ for $n \geq 4$. Let $u$ and $v$ be any two distinct vertices of $L T Q_{n}$. Lemma 3 ensures that there exists a path of $L T Q_{n}$ joining $u$ and $v$ with every length from $d_{L T Q_{n}}(u, v)+2$ to $2^{n}-1$. Since the diameter of $L T Q_{n}$ is $n-1$ for $n=4$ and $\left\lceil\frac{n+3}{2}\right\rceil$ for $n \geq 5$, we discuss this problem based on the assumption that $u$ and $v$ are contained in cycles $C$ of various feasible lengths in $L T Q_{n}$ and 
$d_{C}(u, v)$ satisfies any integer from $D\left(L T Q_{n}\right)+2$ to $2^{n-1}$. The following theorems describe the main result of this paper.

Theorem 1: Let $u$ and $v$ be any two distinct vertices of $L T Q_{4}$. Then, for any integer $m$ satisfying $D\left(L T Q_{4}\right)+2 \leq$ $m \leq 8$, there exists a cycle $C$ of $L T Q_{4}$ containing $u$ and $v$ such that $d_{C}(u, v)=m$ and $2 m \leq l(C) \leq 16$.

Proof. Since $D\left(\mathrm{LTQ}_{4}\right)=3$, we have that $5 \leq m \leq 8$. The correctness of this theorem is verified by our computer program [4].

Theorem 2: Let $u$ and $v$ be any two distinct vertices of $L T Q_{n}, n \geq 5$. Then, for any integer $m$ satisfying $D\left(L T Q_{n}\right)+$ $2 \leq m \leq 2^{n-1}$, there exists a cycle $C$ of $L T Q_{n}$ containing $u$ and $v$ such that $d_{C}(u, v)=m$, where (i) $2 m+1 \leq l(C) \leq 2^{n}$ if $m=D\left(L T Q_{n}\right)+2$ and $n$ is odd, and (ii) $2 m \leq l(C) \leq 2^{n}$ otherwise.

Proof. Since $n \geq 5$, we have that $D\left(L T Q_{n}\right)=\left\lceil\frac{n+3}{2}\right\rceil$. We prove this theorem by induction. By our computer program [4], the correctness of the induction base $L T Q_{5}$ is verified. Assuming that this theorem is true for any $L T Q_{k}$, $5 \leq k \leq n-1$, we show that this theorem is also true for $L T Q_{n}$. Without loss of generality, assume $u$ is in $L T Q_{n-1}^{0}$. Consider the following cases.

Case 1. $v$ is in $L T Q_{n-1}^{1}$ and $u$ is not adjacent to $v$. By Lemma 2, we have that $d_{L T Q_{n}}\left(u,(v)^{n}\right)=d_{L T Q_{n}}(u, v)-1$ or $d_{L T Q_{n}}\left((u)^{n}, v\right)=d_{L T Q_{n}}(u, v)-1$. Without loss of generality, assume $d_{L T Q_{n}}\left((u)^{n}, v\right)=d_{L T Q_{n}}(u, v)-1$. Since $D\left(L T Q_{n}\right)=$ $\left\lceil\frac{n+3}{2}\right\rceil, d_{L T Q_{n}}\left((u)^{n}, v\right) \leq\left\lceil\frac{n+3}{2}\right\rceil-1$. In $L T Q_{n-1}^{1}$, by Lemma 4 , there exists a Hamiltonian path $S$ such that $S(1)=(u)^{n}$ and $d_{S}\left((u)^{n}, v\right)=m-1$, where $\left\lceil\frac{n+3}{2}\right\rceil+2 \leq m \leq 2^{n-1}$. We can write $S$ as $\left[(u)^{n}, S_{1}, v, S_{2}, z\right]$ for some vertex $z$ with $l\left(S_{1}\right)=m-1$, and $S_{2}$ can be written as $\left[v, S_{2}^{1}, x, S_{2}^{2}, z\right]$ for some vertex $x$. Note that $x=v$ if $l\left(S_{2}^{1}\right)=0, x=z$ if $l\left(S_{2}^{1}\right)=2^{n-1}-m$, and $x=v=z$ if both $l\left(S_{1}\right)=2^{n-1}-1$ and $l\left(S_{2}^{1}\right)=0$. For $L T Q_{n-1}^{0}$, distinguish the following subcases.

Subcase 1.1. $n$ is even. Then, $\left\lceil\frac{(n-1)+3}{2}\right\rceil+2=\left\lceil\frac{n+3}{2}\right\rceil+$ 1. By Lemma $3, L T Q_{n-1}^{0}$ has a path $R$ joining $u$ and $(x)^{n}$ with $m-1 \leq l(R) \leq 2^{n-1}-1$. We set $C=$ $\left[u,(u)^{n}, S_{1}, v, S_{2}^{1}, x,(x)^{n}, R, u\right]$, and $l(C)=l(R)+l\left(S_{1}\right)+$ $l\left(S_{2}^{1}\right)+2$. It is easy to obtain that $d_{C}(u, v)=d_{S}\left((u)^{n}, v\right)+1=$ $m$ and $2 m \leq l(C) \leq 2^{n}$. See Fig. 3 for illustration.

Subcase 1.2. $n$ is odd. Then, $\left\lceil\frac{(n-1)+3}{2}\right\rceil+2=\left\lceil\frac{n+3}{2}\right\rceil+2$. Consider the following conditions.

Condition 1.2.1. $m=\left\lceil\frac{n+3}{2}\right\rceil+2$. In $L T Q_{n-1}^{0}$, Lemma 3 ensures that there exists a path $R$ joining $u$ and $(x)^{n}$ with $m \leq l(R) \leq 2^{n-1}-1$. By setting $C=\left[u,(u)^{n}, S_{1}, v, S_{2}^{1}, x\right.$, $\left.(x)^{n}, R, u\right]$, we have that $l(C)=l(R)+l\left(S_{1}\right)+l\left(S_{2}^{1}\right)+2$. Then, $d_{C}(u, v)=m$ and $2 m+1 \leq l(C) \leq 2^{n}$. Figure 3 also illustrates this subcase.

Condition 1.2.2. $\left\lceil\frac{n+3}{2}\right\rceil+3 \leq m \leq 2^{n-1}$. By Lemma 3, there exists a path $R$ of $L T Q_{n-1}^{0}$ joining $u$ and $(x)^{n}$ with $m-1 \leq$ $l(R) \leq 2^{n-1}-1$. We set $C=\left[u,(u)^{n}, S_{1}, v, S_{2}^{1}, x,(x)^{n}, R, u\right]$, then $d_{C}(u, v)=m$ and $2 m \leq l(C) \leq 2^{n}$.

Case 2. $v$ is in $L T Q_{n-1}^{1}$ and $u v$ is an edge of $L T Q_{n}$. Consider the following subcases.

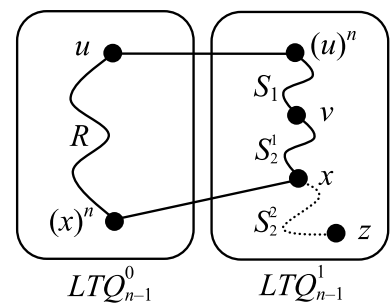

Fig. 3 Case 1 in the proof of Theorem 2. (A straight line represents an edge, and a curve represents a path.)

Subcase 2.1. $\left\lceil\frac{n+3}{2}\right\rceil+2 \leq m \leq 2^{n-2}$ and $2 m \leq l(C) \leq 2^{n-1}$. Let $x=(u)^{2}$ and $y=(u)^{3}$. By Lemma 1 , vertices $u, x,(x)^{n}$, $v$ form a cycle of length 4 , and so do vertices $u, y,(y)^{n}$, and $v$. In $L T Q_{n-1}^{1}-\left\{(y)^{n}\right\}$, by Lemma 6 , there exists a path $S$ joining $v$ and $(x)^{n}$ with $5 \leq l(S) \leq 2^{n-1}-2$. Since $\left\lceil\frac{n+3}{2}\right\rceil \geq 5$ for $n \geq 6$, path $S$ also satisfies $\left\lceil\frac{n+3}{2}\right\rceil \leq l(S) \leq 2^{n-2}-2$, and we can let $l(S)=m-2$. Also by Lemma $6, L T Q_{n-1}^{0}-$ $\{x\}$ has a path $R$ joining $u$ and $y$ with $5 \leq l(R) \leq 2^{n-1}-2$ which also satisfies $m-2 \leq l(R) \leq 2^{n-1}-m-2$. We set $C=\left[u, x,(x)^{n}, S, v,(y)^{n}, y, R, u\right]$, then $d_{C}(u, v)=l(S)+2$ and $l(C)=l(R)+l(S)+4$. Since $l(S)=m-2$ and $m-2 \leq$ $l(R) \leq 2^{n-1}-m-2$, we obtain that $d_{C}(u, v)=m$ and $2 m \leq$ $l(C) \leq 2^{n-1}$ no matter $n$ is odd or even. This result certainly satisfies $2 m+1 \leq l(C) \leq 2^{n-1}$ for $m=\left\lceil\frac{n+3}{2}\right\rceil+2$ with $n$ odd. The illustration of Subcase 2.1 is shown in Fig. 4 (a).

Subcase 2.2. $\left\lceil\frac{n+3}{2}\right\rceil+2 \leq m \leq 2^{n-2}$ and $2^{n-1}+1 \leq l(C) \leq 2^{n}$. Let $x=(u)^{2}$. By Lemma 1 , vertices $u, x,(x)^{n}, v$ form a cycle of length 4 . In $L T Q_{n-1}^{1}, d_{L T Q_{n-1}^{1}}\left((x)^{n}, v\right)=1$, and there exists a Hamiltonian path $S$ by Lemma 4 such that $S(1)=(x)^{n}$ and $d_{S}\left((x)^{n}, v\right)=m-2$. We write $S$ as $\left[(x)^{n}, S_{1}, v, S_{2}, z\right]$ for some vertex $z$, and $S_{2}$ can be rewritten as $\left[v, S_{2}^{1}, y, S_{2}^{2}, z\right]$ for some vertex $y \neq v$. Note that $y=z$ if $l\left(S_{2}^{1}\right)=2^{n-1}-m+1$. In $L T Q_{n-1}^{0}$, by Lemma 7, there exists a path $R$ of $L T Q_{n-1}^{0}-\{x\}$ joining $u$ and $(y)^{n}$ with $2^{n-1}-m-1 \leq l(R) \leq 2^{n-1}-2$. We set $C=\left[u, x,(x)^{n}, S_{1}, v, S_{2}^{1}, y,(y)^{n}, R, u\right]$, then $d_{C}(u, v)=$ $l\left(S_{1}\right)+2$ and $l(C)=l(R)+l\left(S_{1}\right)+l\left(S_{2}^{1}\right)+3$. Since $l\left(S_{1}\right)=m-2$ and $1 \leq l\left(S_{2}^{1}\right) \leq 2^{n-1}-m+1$, we have that $d_{C}(u, v)=m$ and $2^{n-1}+1 \leq l(C) \leq 2^{n}$. The illustration of Subcase 2.2 is shown in Fig. 4 (b).

Subcase 2.3. $2^{n-2}+1 \leq m \leq 2^{n-1}$ and $2 m \leq l(C) \leq 2^{n}$. As in Subcase 2.2, we can find a Hamiltonian path $S=$ $\left[(x)^{n}, S_{1}, v, S_{2}^{1}, y, S_{2}^{2}, z\right]$ in $L T Q_{n-1}^{1}$ such that $d_{S}\left((x)^{n}, v\right)=$ $l\left(S_{1}\right)=m-2$ and $1 \leq l\left(S_{2}^{1}\right) \leq 2^{n-1}-m+1$. In $L T Q_{n-1}^{0}$, by Lemma 7, there exists a path $R$ of $L T Q_{n-1}^{0}-\{x\}$ joining $u$ and $(y)^{n}$ with $m-2 \leq l(R) \leq 2^{n-1}-2$. By setting $C=\left[u, x,(x)^{n}, S_{1}, v, S_{2}^{1}, y,(y)^{n}, R, u\right]$, we have that $d_{C}(u, v)=l\left(S_{1}\right)+2$ and $l(C)=l(R)+l\left(S_{1}\right)+l\left(S_{2}^{1}\right)+3$. It is obvious that $d_{C}(u, v)=m$ and $2 m \leq l(C) \leq 2^{n}$. Figure 4 (b) also illustrates this subcase.

Case 3. $v$ is in $L T Q_{n-1}^{0}$. The following subcases are distinguished.

Subcase 3.1. $\left\lceil\frac{n+3}{2}\right\rceil+2 \leq m \leq 2^{n-2}$ and $2 m \leq l(C) \leq 2^{n-1}$. If $n$ is odd, then $n-1$ is even, and $\left\lceil\frac{(n-1)+3}{2}\right\rceil+2=\left\lceil\frac{n+3}{2}\right\rceil+2$. By the inductive hypothesis, there exists a cycle $C$ of $L T Q_{n-1}^{0}$ 


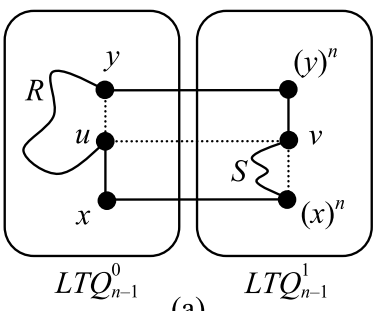

(a)

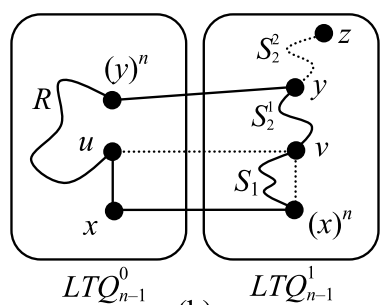

(b)
Fig. 4 Case 2 in the proof of Theorem 2. (A straight line represents an edge, and a curve represents a path.)

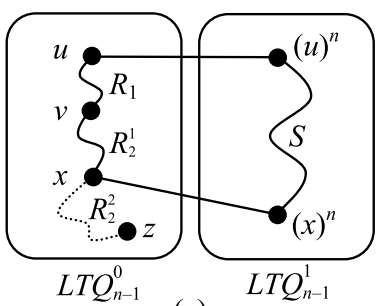

(a)

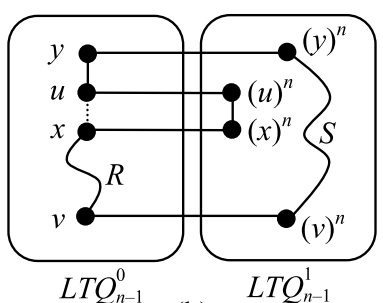

(b)
Fig. 5 Case 3 in the proof of Theorem 2. (A straight line represents an edge, and a curve represents a path.)

containing $u$ and $v$ such that $d_{C}(u, v)=m$ and $2 m \leq l(C) \leq$ $2^{n-1}$. If $n$ is even, then $n-1$ is odd, and $\left\lceil\frac{(n-1)+3}{2}\right\rceil+2=$ $\left\lceil\frac{n+3}{2}\right\rceil+1$. Also by the inductive hypothesis, there exists a cycle $C$ of $L T Q_{n-1}^{0}$ containing $u$ and $v$ such that $d_{C}(u, v)=m$ and $2 m \leq l(C) \leq 2^{n-1}$. Thus, we can find our required cycles in $L T Q_{n}$. Since $\left\lceil\frac{n+3}{2}\right\rceil+2 \leq m \leq 2^{n-2}$ and $2 m \leq l(C) \leq 2^{n-1}$ no matter $n$ is odd or even, this result satisfies $2 m+1 \leq$ $l(C) \leq 2^{n-1}$ for $m=\left\lceil\frac{n+3}{2}\right\rceil+2$ with $n$ odd.

Subcase 3.2. $\left\lceil\frac{n+3}{2}\right\rceil+2 \leq m \leq 2^{n-2}$ and $2^{n-1}+1 \leq l(C) \leq 2^{n}$. By Lemma 4, we have a Hamiltonian path $R$ of $L T Q_{n-1}^{0}$ such that $R(1)=u$ and $d_{R}(u, v)=m$. We write $R$ as $\left[u, R_{1}, v, R_{2}, z\right]$ for some vertex $z$, where $l\left(R_{1}\right)=m$. For convenience, $R_{2}$ can be written as $\left[v, R_{2}^{1}, x, R_{2}^{2}, z\right]$ for some vertex $x$. Note that $x=v$ if $l\left(R_{2}^{1}\right)=0$ and $x=z$ if $l\left(R_{2}^{1}\right)=2^{n-1}-m-1$. In $L T Q_{n-1}^{1}$, by Lemma 3, there exists a path $S$ joining $(u)^{n}$ and $(x)^{n}$ with $2^{n-1}-m-1 \leq l(S) \leq 2^{n-1}-1$. We set $C=\left[u, R_{1}, v, R_{2}^{1}, x,(x)^{n}, S,(u)^{n}, u\right]$, and $l(C)=l\left(R_{1}\right)+l\left(R_{2}^{1}\right)+$ $l(S)+2$. Since $0 \leq l\left(R_{2}^{1}\right) \leq 2^{n-1}-m-1$ and $2^{n-1}-m-1 \leq$ $l(S) \leq 2^{n-1}-1$, we can obtain that $2^{n-1}+1 \leq l(C) \leq 2^{n}$. Thus, $C$ is our required cycle. Figure 5 (a) illustrates this subcase.

Subcase 3.3. $2^{n-2}+1 \leq m \leq 2^{n-1}-1$ and $2 m \leq l(C) \leq$ $2^{n}$. As in Subcase 3.2, we can find a Hamiltonian path $R=$ $\left[u, R_{1}, v, R_{2}^{1}, x, R_{2}^{2}, z\right]$ of $L T Q_{n-1}^{0}$ such that $d_{R}(u, v)=l\left(R_{1}\right)=$ $m$ and $0 \leq l\left(R_{2}^{1}\right) \leq 2^{n-1}-m-1$. In $L T Q_{n-1}^{1}$, Lemma 3 ensures that there exists a path $S$ joining $(x)^{n}$ and $(u)^{n}$ with $m-2 \leq$ $l(S) \leq 2^{n-1}-1$. We set $C=\left[u, R_{1}, v, R_{2}^{1}, x,(x)^{n}, S,(u)^{n}, u\right]$, and $l(C)=l\left(R_{1}\right)+l\left(R_{2}^{1}\right)+l(S)+2$. Then, we can obtain that $2 m \leq l(C) \leq 2^{n}$. Figure 5 (a) also illustrates this subcase.

Subcase 3.4. $m=2^{n-1}$ and $l(C)=2^{n}$. Let $x$ and $y$ be two distinct neighbors of $u$ in $L T Q_{n-1}^{0}-\{v\}$. By Lemma 5, there exists a Hamiltonian path $R$ in $L T Q_{n-1}^{0}-\{u, y\}$ joining $x$ and $v$ with $l(R)=2^{n-1}-3$. Also by Lemma 5 , there exists a Hamiltonian path $S$ in $L T Q_{n-1}^{1}-\left\{(u)^{n},(x)^{n}\right\}$ joining $(y)^{n}$ and $(v)^{n}$ with $l(S)=2^{n-1}-3$. We set $C=\left[u,(u)^{n},(x)^{n}, x\right.$, $\left.R, v,(v)^{n}, S,(y)^{n}, y, u\right]$, and we obtain that $d_{C}(u, v)=l(R)+$ $3=2^{n-1}=m$ and $l(C)=l(R)+l(S)+6=2^{n}$. This subcase is illustrated in Fig. 5 (b).

The above completes the proof.

\section{Concluding Remarks}

Cycles and paths are two fundamental network topologies for parallel and distributed computing. If cycles (paths) of different lengths can be embedded, we can adjust the number of simulated processors and increase the flexibility of demand [11]. Since the panpositionable pancyclicity problem combines cycle embedding with path embedding of various lengths, this problem supports extremely flexible cycle embedding. In this paper, we demonstrate that locally twisted cubes $L T Q_{n}, n \geq 4$, are panpositionably pancyclic. Precisely, for any two distinct vertices $u$ and $v$ of $L T Q_{n}$ and for any integer $m$ with $D\left(L T Q_{n}\right)+2 \leq m \leq 2^{n-1}$, we can find a cycle $C$ containing $u$ and $v$ such that $d_{C}(u, v)=m$, and the length $l(C)$ of cycle $C$ satisfies (i) $2 m+1 \leq l(C) \leq 2^{n}$ if $m=D\left(L T Q_{n}\right)+2$ and $n$ is odd, and (ii) $2 m \leq l(C) \leq 2^{n}$ otherwise. This result improves on the previous one that locally twisted cubes are panpositionably Hamiltonian, where any two distinct vertices can be positioned with a given distance only on a Hamiltonian cycle. This paper indicates that in locally twisted cubes, the cycle embedding containing any two vertices with a feasible distance is extremely flexible. For a future work, we are motivated to investigate if the panpositionable pancyclicity of locally twisted cubes can be $d_{L T Q_{n}}(u, v)+2 \leq m \leq 2^{n-1}$ with $2 m \leq l(C) \leq 2^{n}$ instead of $D\left(L T Q_{n}\right)+2 \leq m \leq 2^{n-1}$.

\section{Acknowledgments}

The author would like to express his gratitude to the anonymous referees and the editor for their valuable suggestions for improving the clarity of this work. This work is supported in part by National Chin-Yi University of Technology under Contract NCUT16-R-MM-017.

\section{References}

[1] Y. Alavi and J.E. Williamson, "Panconnected graphs," Studia Scientiarum Mathematicarum Hungarica, vol.10, pp.19-22, 1975.

[2] J.A. Bondy, "Pancyclic graphs," Journal of Combinatorial Theory-Series B, vol.11, no.1, pp.80-84, Aug. 1971. DOI: 10.1016/00958956(71)90016-5

[3] H.-C. Chan, J.-M. Chang, Y.-L. Wang, and S.-J. Horng, "Geodesicpancyclicity and fault-tolerant panconnectivity of augmented cubes," Applied Mathematics and Computation, vol.207, no.2, pp.333-339, Jan. 2009. DOI: 10.1016/j.amc.2008.10.061

[4] H.-C. Chen, "A data verification report on the panpositionable pancyclicity of locally twisted cubes," Available at http://mis.web2.ncut. edu.tw/ezfiles/19/1019/img/1258/CHENHC3.htm

[5] J. Fan, X. Lin, and X. Jia, "Optimal path embeddings in crossed cubes," IEEE Trans. Parallel Distrib. Syst., vol.16, no.12, 
pp.1190-1200, Oct. 2005. DOI: 10.1109/TPDS.2005.151

[6] M.C. Golumbic, Algorithmic Graph Theory and Perfect Graphs, Academic Press, New York, 1980.

[7] K.S. Hu, S.-S. Yeoh, C. Chen, and L.-H. Hsu, "Node-pancyclicity and edge-pancyclicity of hypercube variants," Information Processing Letters, vol.102, no.1, pp.1-7, April 2007. DOI: 10.1016/j.ipl. 2006.10.008

[8] T.-L. Kung, "Flexible cycle embedding in the locally twisted cube with nodes positioned at any prescribed distance," Information Sciences, vol.242, pp.92-102, Sept. 2013. DOI: 10.1016/j.ins.2013.04. 029

[9] T.-L. Kung and H.-C. Chen, "Improving the panconnectedness property of locally twisted cubes," International Journal of Computer Mathematics, vol.91, no.9, pp.1863-1873, Jan. 2014. DOI: $10.1080 / 00207160.2013 .860448$

[10] T.-L. Kung, C.-K. Lin, T. Liang, L.-H. Hsu, and J.J.M. Tan, "On the bipanpositionable bipanconnectedness of hypercubes," Theoretical Computer Science, vol.410, no.8-10, pp.801-811, March 2009. DOI: $10.1016 /$ j.tcs.2008.11.004

[11] T.-J. Lin, S.-Y. Hsieh, and J.S.-T. Juan, "Embedding cycles and paths in product networks and their applications to multiprocessor systems," IEEE Trans. Parallel Distrib. Syst., vol.23, no.6, pp.1081-1089, June 2012. DOI: 10.1109/TPDS.2011.245

[12] M. Ma and J.-M. Xu, "Panconnectivity of locally twisted cubes," Applied Mathematics Letters, vol.19, no.7, pp.673-677, July 2006. DOI: 10.1016/j.aml.2005.08.021

[13] J.-H. Park, H.-S. Lim, and H.-C. Kim, "Panconnectivity and pancyclicity of hypercube-like interconnection networks with faulty elements," Theoretical Computer Science, vol.377, no.1-3, pp.170-180, May 2007. DOI: 10.1016/j.tcs.2007.02.029

[14] Y.-H. Teng, J.J.M. Tan, and L.-H. Hsu, "Panpositionable hamiltonicity and panconnectivity of the arrangement graphs," Applied Mathematics and Computation, vol.198, no.1, pp.414-432, April 2008. DOI: 10.1016/j.amc.2007.08.073

[15] X. Xu, W. Zhai, J.-M. Xu, A. Deng, and Y. Yang, "Fault-tolerant edge-pancyclicity of locally twisted cubes," Information Sciences, vol.181, no.11, pp.2268-2277, June 2011. DOI: 10.1016/j.ins.2011. 01.031

[16] X. Yang, D.J. Evans, and G.M. Megson, "The locally twisted cubes," International Journal of Computer Mathematics, vol.82, no.4, pp.401-413, 2005. DOI: 10.1080/0020716042000301752

[17] X. Yang, G.M. Megson, and D.J. Evans, "Locally twisted cubes are 4-pancyclic," Applied Mathematics Letters, vol.17, no.8, pp.919-925, Aug. 2004. DOI: 10.1016/j.aml.2003.10.009

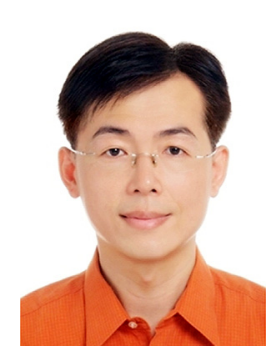

Hon-Chan Chen received the BS, MS, and $\mathrm{PhD}$ degrees in information management from National Taiwan University of Science and Technology, Taipei, Taiwan, in 1992, 1994, and 1998. From 2000 to 2002, he was an assistant professor in National Kaohsiung Marine University, Kaohsiung, Taiwan. Since 2002, he has been teaching in the Department of Information Management, National Chin-Yi University of Technology, Taichung, Taiwan. He is currently an associate professor. His research interests include algorithms, parallel processing, and graph theory. 